\title{
Allocation of Energy Efficient Sub-Channel in Cognitive Radio Networks for Television Systems
}

\author{
P.N.V.S.N. Murthy ${ }^{1}$, S. Vidya Sagar Appaji ${ }^{2}$ \\ M. Tech, CSE Department, MVGR College of Engineering, Vizianagaram, India ${ }^{1}$ \\ Assistant Professor, CSE Department, MVGR College of Engineering, Vizianagaram, India ${ }^{2}$
}

\begin{abstract}
The cognitive radios those are energy efficient which are operating in the television systems that means in the television white spaces where the vacant frequencies available compares with the other cognitive radio devices because of the interference constraint which is for whole frequency band rather than per signal carrier. In this paper we considered the Ratefair channel assignment protocol which is explained in the energy efficient frequency and power allocation for cognitive radios in television systems, taken from IEEE systems journal. In this protocol the users are allocated with the sub channels which are energy efficient based on their transmission rates. Users are arranged in the descending order based on the transmission rates. The user with higher transmission rate is assigned with the sub channel on which the user obtains a larger gain. But at the same time lower transmission rate user may also get larger gain on the same sub channel which is already allocated to the higher transmission rate. Because of the low transmission rate the user cannot acquire the sub channel on which the gain is large. Low transmission rate is may be due to larger separation distance between the cognitive base station or may be because of the more interference by the secondary users. In this paper we use two algorithms, one is recursive algorithm which is used to find the shortest distance and also the most reliable path by calculating the packet loss rate and another algorithm is Dynamic threshold algorithm which is used to reduce the interference of the secondary users. As a result transmission rate of users increases, delay decreases, data will be transmitted more reliably. Simulations are compared with the existing protocol and showed that the proposed method increases the transmission rate and decreases the delay in transmission and the channels are allocated which are more Energy Efficient than the existing.
\end{abstract}

Keywords: cognitive radio, channel assignment, TV white spaces, energy efficiency.

\section{INTRODUCTION}

Cognitive Radio Networks in Television Systems consists of the TV white spaces. These Television White spaces are the vacant frequencies which are not used by the primary or licensed users in the available spectrum are made available to the secondary or unlicensed users for usage. Television Frequencies changes from one region to the another region. According to the IEEE 802.22 these TV frequencies are operates on very high range. Every region has some interference constraint in order to control the interference of the secondary users with the primary users spectrum. Therefore these frequencies to the primary users are allocated by assigning the sub channels to the primary users from the cognitive base station. The sub channels that are allocated to the users should be energy efficient.

So in our project we assign the sub channels and allocates the resources such as the frequencies to the users from the cognitive base station to the primary or the secondary users. In this we considered the channel assignment protocol known as Ratefair channel assignment protocol. This protocol explains that the sub channels are allocated to the primary users based on the users transmission rates. This protocol consists of two stages that will be described below. In the first stage the all the available users calculates their transmission rates for the downlink transmissions that is from cognitive base station to the primary users. As it is the downlink transmissions the transmissions rates of all the available users are known by the cognitive base station. After the transmission rates of all the users are known the users are arranged in descending order based on their transmission rates. That is the user with higher transmission rate is first and lower transmission rates is at last. Now the user with the higher transmission rate is allowed to select the sub channel from the available sub channels in that region or spectrum. The user is assigned with a sub channel on which the gain is more when compared with all the available sub channels.

In the second stage the users checks that on the assigned sub channel the gain obtained is more or less. If the gain is more when compared to other available sub channels then that sub channel is allocated to that particular primary user. And then the allocated sub channel is removed from the available sub channel list. Now from the remaining available sub channels the next higher transmission rate user is assigned a sub channel on which that particular user has the maximum gain. In this protocol we found that because of the lower transmission rates, some users cannot acquire the 
Vol. 6, Issue 6, June 2017

sub channels on which the respective user is experiencing a maximum gain that is the signal strength is good and the data transmission is not that much reliable because of this lower transmission rate.

So in this protocol the problem is that because of lower transmission rates the users cannot acquire the channels on which they get larger gain but all the primary users are equally paid for their data transmissions. Therefore in our project we proposed three modules to overcome these issues to increase the transmission rates of the primary users and as a result it reduces the time delay, network congestion, data load on cognitive base station which will be energy efficient. The lower transmission rates are may be due to large separation distance between the primary user and the cognitive base station or another reason may be due to higher interference of the secondary users in the spectrum in the presence of the primary users. We use the recursive algorithm for finding the shortest distance between the user and the cognitive base station and also calculates the packet loss on that path. Dynamic threshold energy algorithm is used to reduce the interference of the secondary users. Mesh topology is created for the reduction of the time delay and the load on the cognitive base station in the downlink transmissions.

\section{RELATED WORK}

Jie Xu , Ling Qui , shunquing zhang concentrated on minimising the Energy Efficiency (EE) for the Multiple Input Multiple Output broadcasting channels, where a power model is taken into account. As the Energy Efficiency of the MIMO broadcasting is non concave, in this paper it is again changed as a quasi concave function which is based on the uplink-downlink duality. After that, block co-ordinate ascent algorithm is used to obtain the minimal transmission policy as efficient as possible, and the results are proved to be convergent. Simulations are created and the results are validated. The simulations of the proposed scheme and the existing scheme are compared on the efficiency of the energy. Block co ordinate ascent algorithm is used in this paper to maximise the energy efficiency. Kandasamy illanko explained the ratefair channel assignment protocol for assigning sub channels for the primary users. This protocol arranges the users in the descending order based on their transmission rates. Higher transmission rate user selects one of the available sub channels on which the signal gain is more among all the available sub channels. He also concentrates on the optimisation of the power allocation for the cognitive radios in television systems. In Xiong et..al., the expanded vitality productivity is subjected to the elements, particularly, low appropriate client sums, full limit constraints, related a transmission medium substance to monitor the principal clients or diverse gadgets that are operational in neighbourhood regions. We tend to look at the issue of the convention there up on of changed and enhanced particular types of continous bolster portions of course of action that builds the vitality proficiency of OFDMA downloads. We offer the good to analyse the exploitation reproduced numerical results and shows that the game plan acquire more energy efficiency in comparision with libbed components of existing conventions. Range usuage can be used uncommonly by permitting it usuable for another client to approach persistently accessible by the main clients at the right zone and the term reaction.

\section{METHODOLOGY}

\section{Dynamic Threshold Algorithm}

Dynamic Threshold Algorithm is used to reduce the interference of the secondary or unlicensed users to the primary or licensed users of the spectrum which is available. Secondary users or unlicensed users have to sense the spectrum continuously for the vacant frequencies at certain locations where the primary users or licensed users are unavailable that means in the absence of the primary users in the available spectrum. Secondary users should use the spectrum or available frequencies in the absence of the primary users in order to reduce the interference for the primary users. Secondary users have the energy detectors which predicts the received signal strength which includes both the noise and the primary user signal. If the threshold value is a fixed value then the energy detectors of the secondary users which senses the spectrum based on the received signal compares that signal strength with the fixed threshold and decides the presence of the primary user in the available spectrum. If the received signal strength is below the fixed threshold value then the secondary user predicts that the primary user is unavailable in the spectrum and if the received signal strength is higher than the fixed threshold value then the secondary user predicts that the primary user is available in the spectrum. But as the received signal strength is comprises of both the primary user signal and the noise , it is not that accurate that the prediction of the secondary user. If noise strength alone in the received signal without the primary user signal is greater than the fixed threshold then the secondary user predicts that the primary user is available in the spectrum and the secondary users won't use the available frequencies in the spectrum. If the noise strength alone is less than the fixed threshold then the secondary user predicts that the primary user is unavailable in the spectrum and uses the spectrum or the frequencies which causes interference or disturbances for the primary users. This false prediction is known as the probability of false alarm. So in order to reduce this interference for the primary users the prediction of the secondary users for the availability of the primary users should be more accurate. Therefore if the threshold value changes dynamically along with the noise signal instead of fixed value the sensing of the secondary 
users will be more accurate. As the noise signal is not constant it changes along with the signal strength based on the variance of the signal the threshold values also changes accordingly.

\section{Recursive Algorithm}

Recursive algorithm is used to reduce the distance between the cognitive base station and the primary users. Transmission delay can be caused due to the large separation distance between the base station and the primary users. This algorithm finds the shortest distance to the primary users from the cognitive base station which is most efficient. In this algorithm the source finds the shortest path to its neighbours first in the path selection process to the destination. It also checks for the path in which it has lowest packet loss ratio while transmitting the data. Based on the packet loss and the distance among the neighbours from the source, the source sets the priority to the neighbours. This algorithm also maintains the partially shared buffer for the priorities. This buffer is partially shared by all the nodes involved in the path selection for the destination. After that the neighbours again initiate the same process for finding the path for the destination. If the neighbours found any path which is more efficient than the lower priority node inthe buffer then that path is replaced with the lower priority path in the buffer. In this way recursive algorithm finds the shortest path along with the lowest packet loss path for the destination. The path in which the packet loss ratio is less and the distance is low then that path is given the highest priority for the transmission. The path which has packet loss is medium and distance is short the that path is set to medium priority in the buffer. The path which has the larger distance to the destination and the packet loss through that path is high then that path is set to the low priority in the partial buffer which is shared among the nodes.

Step 1. Create a Network with N number of nodes in ns2

Step 2. Identify the Source Node ' $S$ ' and Destination Node ' $D$ '

Step 3. Set Current Node $==$ Source as the Current Node

Step 4. While Current Node is less than the Destination Node

Step 5. Define the neighbouring nodes of the current node that is for source

Neighbour(1),Neighbour(2)......Neighbour(M)

Step 6. For loop where $\mathrm{i}=1$ to $\mathrm{M}$

Step 7. Calculate the parameter which defines the packet loss and the data rate

Step 8. [Sender End calculation Logic]

Step 9. Calculate these rules under the calculation process

Step 10. If ( calculate(Packet Loss rate(Neighbour(i),Low) and calculate(Data rate(Neighbour(i),High)

Step 11. Set the Priority of that node as (Neighbour(i))=High)

Step 12. Else If ( calculate(Packet Loss rate(Neighbour(i)),Medium) and calculate(Data rate(Neighbour(i)),Medium)

Step 13. Set Priority of that node as medium (Neighbour(i))=Medium)

Step 14. Else If ( calculate(Packet Loss rate(Neighbour(i)),Low) and calculate(Data rate(Neighbour(i)),Low)

Step 15. Set Priority of that particular node as(Neighbour(i))=Low.(black hole node found)

Step 16. Define the node which has the highest priority which we call as temp

Step 17. Set Current Node=temp

\section{PROPOSED SYSTEM}

\section{Network Formation}

In the network formation we deployed fifty nodes in the network simulator window. Among those nodes one is selected as source that means cognitive base station and three destinations are used as the primary users. According to the Ratefair channel assignment protocol that is used in the existing the users with transmission rates are arranged in the descending order. The users with higher transmission rate acquires the channel from the available sub channel on which the user gets the larger gain. But at the same time the lower transmission rate user may also get the larger gain on already allocated channel to the higher transmission rate user. Due to this lower transmission the user experiencing delay in the transmission from the cognitive base station. So we uses secondary user as temporary primary user for forwarding the data to the destination to reduce the delay and increase the transmission rate.

\section{Resource Allocation Optimization Problem}

In this project the resource allocation means allocation of the channel and the allocation of the frequency among the users in the simulation we have created using the network simulator tool. We mainly concentrates on how to minimise or optimise the allocation of the resources such as frequencies or channels or bandwidth. If the base station has to send the data for transmission to the primary users it has to send all the available users individually which causes a lot of time and lots of energy will be consumed. So we have to minimise the energy utilisation of the primary users. As the frequency ranges of the televisions are changes form one region to another region the bandwidth should not be constant. It is measured in hertz. 
So in order to reduce or minimise the resource allocation problem the cognitive base station should limit its downlink transmission. Instead of transmitting the data to all the users individually from the cognitive base station if the data is transmitted to only one user in particular region and from that user the data is transmitted to all the users in that regions. That means here we are using the forwarders to transmit the data to the users of the particular region. The forwarders receive the data that is transmitted by the cognitive base station and transmits the data to all the primary users present in that certain region. Mesh network is created in the simulation such that if any one communication link is down the will be transmitted through the another communication link without any delay and also the users are formed into clusters with a cluster head. Here we call the cluster head as the forwarders in our project. Due to this the time delay for the downlink transmission is reduced and also the load on the cognitive base station for the transmission is reduced. Now the cognitive base station sends the data to the particular region forwarder and the forwarder transmits the data to the destination. The congestion or traffic in the network is reduced as the load of transmission is reduced by the creation of the mesh network topology. In this way we can reduce the allocation of the resources to the primary users from the cognitive base station and as a result the traffic in the network, load on the base station and the time delay are minimised which says that the energy is efficient.

\section{Sub Channel Allocation Protocol}

In the existing the sub channel allocation protocol known as Ratefair channel assignment protocol is used. In this protocol the available users calculates the downlink transmission rates from the cognitive base station to the primary users. After calculation of the user transmission rates, based on their transmission rates all the available users are arranged in descending order. From the available sub channels the user with the higher transmission rate among all the users is allocated a sub channel from the available sub channels in that spectrum area on which the user gets the higher gain means good signal strength such that the data transmission will be more reliable. After that the user calculates the interference constraint of that sub channel. This protocol involve two stages. In the first stage the above mentioned process is carried out. In the second stage again the user checks whether on the allocated channel the gain is maximum or not when compared with the available sub channels. And then the allocated sub channel is removed from the available sub channels. Now the next higher transmission rate user is allocated the sub channel on which the user has a larger gain from the remaining available sub channels. In this way the sub channels are allocated using the Ratefair channel assignment protocol in the existing. But due to this assignment the lower transmission rate user which may have larger gain on the already allocated sub channel to the higher transmission rate user. It also reduces the transmission and causes transmission delay for the lower transmission rate users and also cause energy delay due to low transmission. So the transmission rates of the users have to be increased in order to allocate channels to the users efficiently. So if we increase the transmission rates of the lower transmission rate users then the sub channels can be assigned to the users on which they get higher gain that means good signal strength such that data will be transmitted more reliably.

\section{RESULTS AND ANALYSIS}

Delay of network or transmission shows the time that is taken to travel the data or packet from the cognitive base station to the primary users.

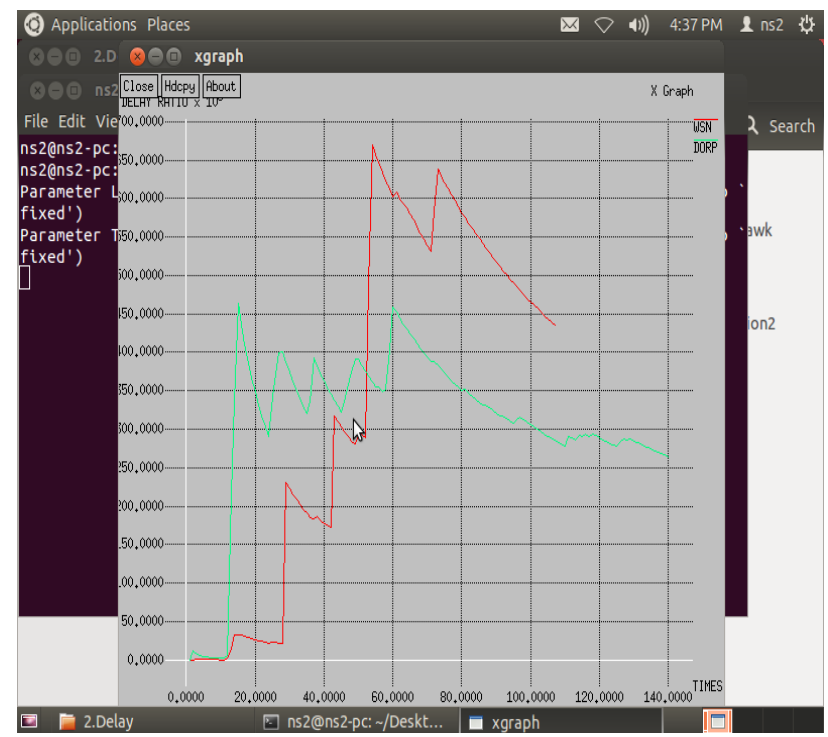

Figure 5.1. Delay 
Measurements such as multiples or fractions of seconds are used to measure the delay of the transmission. Depending on the position of the nodes which are communicating, delay also changes according to the position of the node as some nodes may die due to energy loss.

Transmission delay is the time or duration that the packet or data takes to transmit the packet's or data bits onto the connection. The time taken to transmit the data or packet through the channel experiences minimum level of delay. Delay may occur due to the heavy traffic or congestion in the networks. The above graph shows that the delay experienced by existing method is huge when compared to DORP.

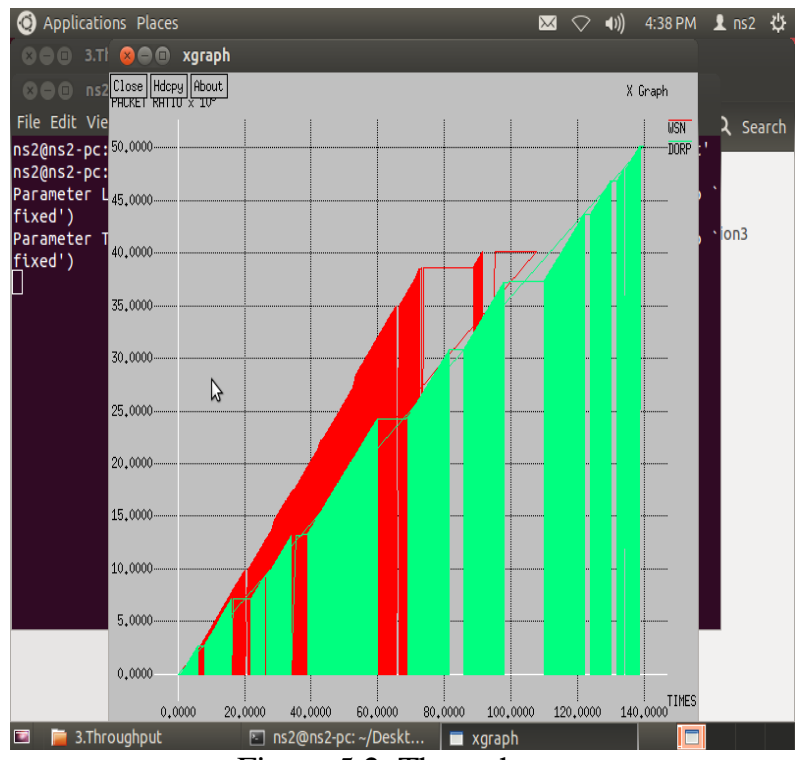

Figure 5.2. Throughput

Packet loss is that the packets which are transmitted from the source to the destination among which are not delivered to the destination successfully. Packet loss is typically caused by network congestion. Packet loss can reduce throughput, due to malfunctioning of network. The channel loss graph above says that the loss ratio that exist between the proposed and existing.

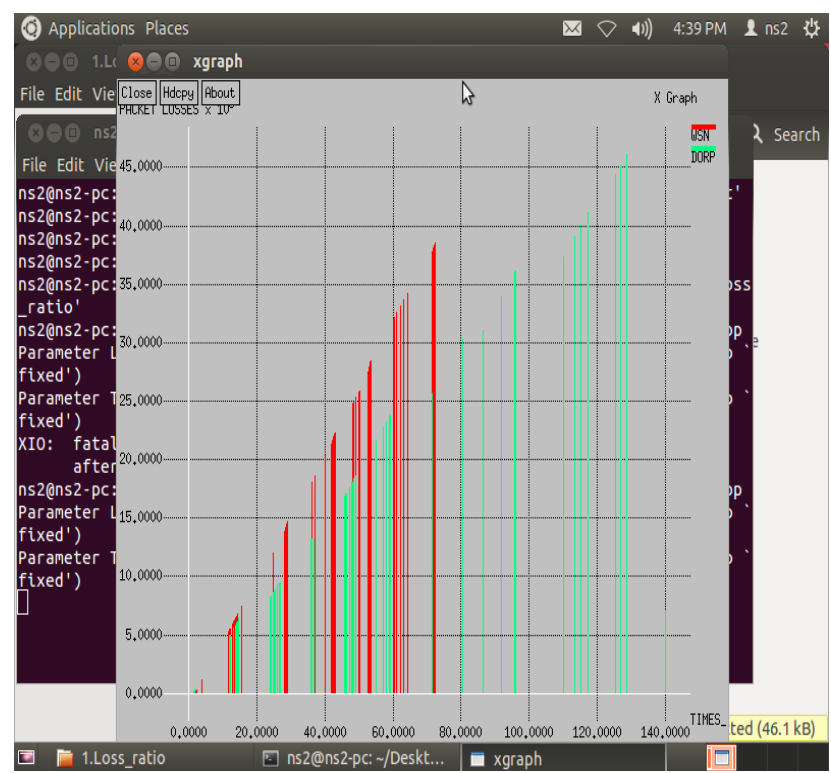

Figure 5.3. Loss Ratio

Channel graphs are widely used in the study of blocking networks. The graph which is obtained as a result for the above simulation shows that DORP based channel allocation of channels are effective when compared to wireless sensor networks. 


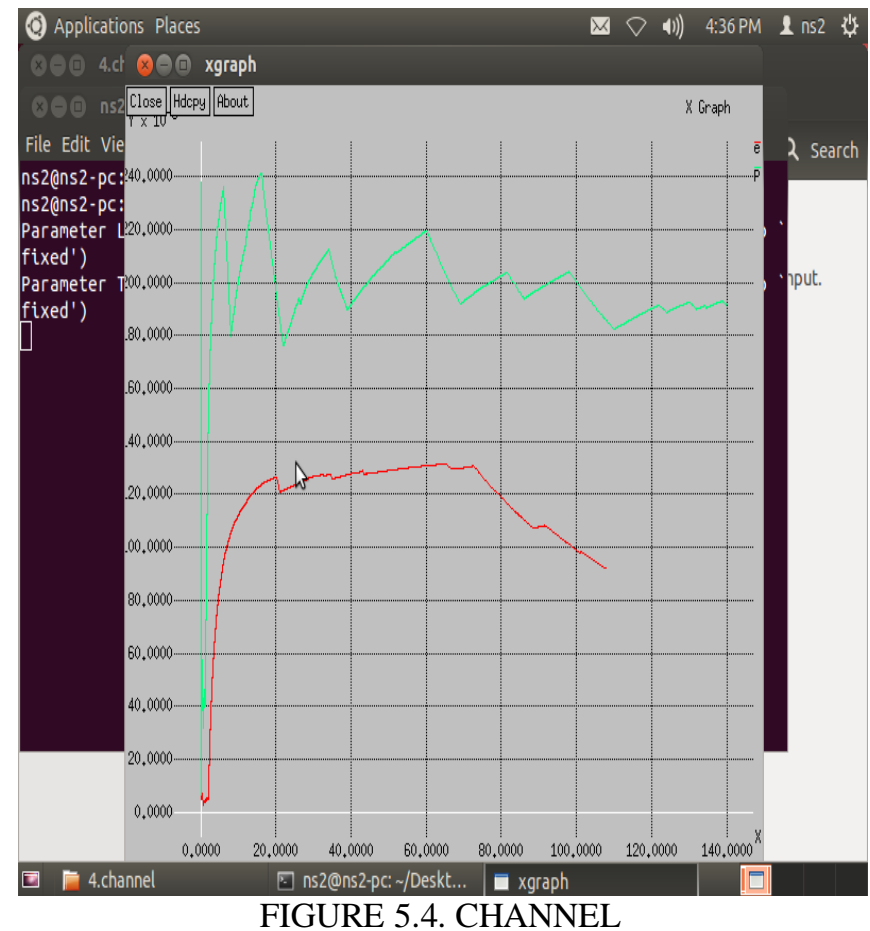

FIGURE 5.4. CHANNEL

\section{CONCLUSION}

In this project channels are allocated to the primary users from the cognitive base station which are more energy efficient. By using the recursive algorithm the delay and the packet loss while transmitting the packet or data is reduced. As the delay is reduced therefore the energy will be consumed efficiently by the nodes and by the reduction of the distance between the cognitive base station and the primary user the transmission rate also increases so that the data will be delivered reliably. By using the dynamic threshold algorithm the interference is reduced such that the throughput is increased. As the interference is reduced the transmission rate of the users also increases such that lower transmission rate users transmission increases such that now they can acquire the channel on which that user has the larger gain. The mesh networks are created in the simulation such that to reduce the time delay for the downlink transmission from base station to the primary users. After that the licenses for all the users are cancelled and then those users for whom the channels are required for them only the channels are allocated and no more primary and secondary users or licensed or unlicensed users. As the time delay is reduced, transmission delay is reduced, transmission rate increased, interference among the primary users and secondary users is reduced we can conclude that the energy is efficient when compared to the existing system.

\section{REFERENCES}

[1] Jie Xu, Ling Qui, shunquing zhang, Energy Efficient Iterative Water filling for the MIMO Broadcasting channels, IEEE Wireless Communications and Networking conference, 2012.

[2] Kandasamy illanko, Alagan Anpalagan, Dimitri Androutsos, Dual Methods for Power Allocation for Radios Coexisting in Unlicensed Spectra, IEEE Communication society and Globecom 2010 proceedings.

[3] Liqun Fu, Ying jun, Jianwei Huang, IEEE journal on selected areas in communications, vol.31, no.11, November 2013.

[4] Yan chen, Oliver Blume, Azeddine gati, Antonio Capone, Chi-en Wu, Tom Martezza, Energy Saving: Scaling Network Energy Efficiency Faster Than Traffic Growth, IEEE WCNC workshop on future green end to end wireless network.

[5] S.Haykin, Cognitive Radio: brain - empowered wireless communications, IEEE journal on selected areas of communications, volume 23, issue: 2,2010 .

[6] Guowang Miao, Nageen Himayat, Geoffrey Ye Li, Energy Efficient link adaptation in frequency selective channels, IEEE Transactions on communications, vol 58, issue:2, feb 2010.

[7] R.S. Prabhu, B.Daneshrad, An Energy efficient water filling algorithm for OFDM Systems, IEEE international conference on communications, 2010.

[8] Ziaul Hasan, Gaurav Bansal, Ekram Hossain, Energy efficient power allocation in OFDM based cognitive radio systems: a risk return model, IEEE Transactions on wireless communications, volume:8, issue:12, 2010.

[9] Carl R.steveson, Gerald chouinard, Zhongding Lei, Wendog Hu, The first cognitive radio wireless regional area network standard, IEEE Standards in communications and networking, 2009.

[10] Anh tuan hoang, Ying chang liang, MD habibul islam, power control and channel allocation in cognitive radio network with primary users cooperation, IEEE transactions on mobile computing, volume:9,issue:3,march 2010.

[11] C. Passiotore, p. camadra, A centralised inter-network resource sharing scheme in IEEE 802.22 cognitive networks, IEEE Adhoc networking workshop, 2011. 